\title{
ON THE COMPOSITION OPERATORS ON BANACH WEIGHTED HARDY SPACES
}

\author{
Fatemeh Zangeneh ${ }^{1}$, Bahmann Yousefi $^{2} \S$ \\ ${ }^{1,2}$ Department of Mathematics \\ Payame Noor University \\ P.O. Box: 19395-3697, Tehran, IRAN
}

\begin{abstract}
In this paper we consider composition operators on weighted Hardy spaces and the aim of the paper is to investigate the numerical range of composition operators.
\end{abstract}

AMS Subject Classification: 47B37, 47A16

Key Words: numerical range, composition operator, compact operator, weak topology

\section{Introduction}

Let $\{\beta(n)\}_{n}$ be a sequence of positive numbers with $\beta(0)=1$ and let $1<p<\infty$. Let $f=\{\hat{f}(n)\}_{n=0}^{\infty}$ be such that

$$
\|f\|_{p}=\|f\|_{H^{p}(\beta)}=\left(\sum_{n=0}^{+\infty}|\hat{f}(n)|^{p} \beta(n)^{p}\right)^{1 / p}<\infty .
$$

The notation $f(z)=\sum_{n=0}^{+\infty} \hat{f}(n) z^{n}$ shall be used weather or not the series converges for any value of $z$. The space of such formal power series is called the weighted Hardy space, which is denoted by $H^{p}(\beta)$. In the case $p=2$, the classical Hardy

Received: $\quad$ March 27, 2017

Revised: July 3, 2017

Published: July 27, 2017

$\S_{\text {Correspondence author }}$ (c) 2017 Academic Publications, Ltd. url: www.acadpubl.eu 
space, Bergman space and the Dirichlet space are weighted Hardy spaces with $\beta(n)=1, \beta(n)=(n+1)^{-\frac{1}{2}}$ and $\beta(n)=(n+1)^{\frac{1}{2}}$, respectively. The spaces $H^{p}(\beta)$ are reflexive Banach spaces with the norm $\|\cdot\|_{p}$ and the dual of $H^{p}(\beta)$ is $H^{q}\left(\beta^{\frac{p}{q}}\right)$ where $\frac{1}{p}+\frac{1}{q}=1$ and $\beta^{\frac{p}{q}}=\left\{\beta(n)^{\frac{p}{q}}\right\}_{n}$.

Recall that for $g(z)=\sum_{n=0}^{\infty} \hat{g}(n) z^{n}$ in $H^{q}\left(\beta^{p / q}\right)$, note that

$$
\|g\|_{q}^{q}=\|g\|_{H^{q}\left(\beta^{p / q}\right)}^{q}=\sum_{n=0}^{\infty}|\hat{g}(n)|^{q} \beta(n)^{p}<\infty .
$$

If $\lim \frac{\beta(n+1)}{\beta(n)}=1$ or $\liminf \beta(n)^{\frac{1}{n}}=1$, then $H^{p}(\beta)$ consists of functions analytic on the open unit disk $U$.

A complex number $\lambda$ is said to be a bounded point evaluation on $H^{p}(\beta)$ if the functional of point evaluation at $\lambda, e_{\lambda}$, is bounded. A complex number $\lambda$ is a bounded point evaluation on $H^{p}(\beta)$ if and only if $\left\{\frac{\lambda^{n}}{\beta(n)}\right\}_{n} \in l^{q}$.

Let $\varphi$ be an analytic self map of $U$. A composition operator $C_{\varphi}$ maps an analytic function $f \in H^{p}(\beta)$ into $\left(C_{\varphi} f\right)(z)=f(\varphi(z))$. The function $\varphi$ is called the composition map. Some sources on formal power series and composition operators include [1-7].

\section{Main Result}

This work represents the necessary and sufficient conditions for the closedness of the numerical range of a compact composition operator acting on weighted Hardy spaces $H^{p}(\beta)$.

In the following we define some definitions that will be used in the main theorem.

Definition 2.1. Let $1<p<\infty$ and $\frac{1}{p}+\frac{1}{q}=1$. Let $f(z)=\sum_{n=0}^{\infty} \hat{f}(n) z^{n} \in$ $H^{p}(\beta)$ and define $f^{*}(z)=\sum_{n=0}^{\infty}|\hat{f}(n)|^{(p-q) / q} \hat{f}(n) z^{n}$.

Note that $\left\|f^{*}\right\|_{q}^{q}=\left\|f^{*}\right\|_{H^{q}\left(\beta^{p / q}\right)}^{q}=\sum_{n=0}^{\infty}|\hat{f}(n)|^{p} \beta(n)^{p}=\|f\|_{p}^{p}$ and obviously one can see that $<f, f^{*}>=\|f\|_{p}^{p}$. Also 
Definition 2.2. Let $1<p<\infty$ and $\frac{1}{p}+\frac{1}{q}=1$. Suppose that $g(z)=$ $\sum_{n=0}^{\infty} \hat{g}(n) z^{n}$ belongs to $H^{q}\left(\beta^{p / q}\right)$ and define ${ }^{*} g(z)=\sum_{n=0}^{\infty}|\hat{g}(n)|^{(q-p) / p} \hat{g}(n) z^{n}$.

Notice that $\left\|^{*} g\right\|_{p}^{p}=\sum_{n=0}^{\infty}|\hat{g}(n)|^{q} \beta(n)^{p}=\|g\|_{q}^{q}<\infty$ and so ${ }^{*} g \in H^{p}(\beta)$. Obviously, one can see that ${ }^{*}\left(f^{*}\right)=f$ for all $f$ in $H^{p}(\beta)$ and $\left({ }^{*} g\right)^{*}=g$ for all $g$ in $\left(H^{p}(\beta)\right)^{*}$. Also, clearly $<g,{ }^{*} g>=\|g\|_{q}^{q}$.

Definition 2.3. If $T$ is a bounded linear operator on $H^{p}(\beta)$, the numerical range of $T$ is denoted by $W(T)$ that is defined by

$$
W(T)=\operatorname{co}\left\{<T f, f^{*}>: f \in H^{p}(\beta) \text { and }\|f\|_{p}=1\right\}
$$

Note that clearly $W(T)=\left\{\left\langle T\left(^{*} g\right), g\right\rangle: g \in\left(H^{p}(\beta)\right)^{*}\right.$ and $\left.\|g\|_{q}=1\right\}$.

Theorem 2.4. Let $\frac{1}{p}+\frac{1}{q}=1, \liminf \beta(n)^{\frac{1}{n}}=1, \sum_{n=0}^{\infty} \frac{1}{\beta(n)^{q}}=\infty$, and $C_{\varphi}$ be compact on $H^{p}(\beta)$. Suppose that there exists $\xi_{0}$ in $\partial U$ such that the limit of ${ }^{*} e_{\lambda}(\varphi(\lambda))$ exists and is finite as $\lambda \rightarrow \xi_{0}$. Then $0 \in W\left(C_{\varphi}\right)$ if and only if $W\left(C_{\varphi}\right)$ is closed.

Proof. First note that since $\lim \inf \beta(n)^{\frac{1}{n}}=1$, thus for each $\lambda$ in the open unit disk, the functional of evaluation at $\lambda, e_{\lambda}$, is a bounded linear functional and we have

$$
e_{\lambda}(z)=\sum_{n=0}^{\infty} \frac{\bar{\lambda}^{n}}{\beta(n)^{p}} z^{n}
$$

and

$$
\left\|e_{\lambda}\right\|^{q}=\sum_{n=0}^{\infty} \frac{|\lambda|^{n q}}{\beta(n)^{q}} .
$$

Now suppose that $\left\{h_{n}\right\}_{n}$ is a sequence of unit vectors in $H^{p}(\beta)$. By weakly compactness of ball $H^{p}(\beta)$, a subsequence of $\left\{h_{n}\right\}_{n}$ is weakly convergent. For simplicity we suppose that $\left\{h_{n}\right\}_{n}$ converges weakly to a vector $h$ in $H^{p}(\beta)$. Then we have

$$
\begin{aligned}
\left|<C_{\varphi} h_{n}, h_{n}^{*}>-<C_{\varphi} h, h^{*}>\right| \leq & <C_{\varphi} h_{n}, h_{n}^{*}>-<C_{\varphi} h, h_{n}^{*}>\mid \\
& +\left|<C_{\varphi} h, h_{n}^{*}>-<C_{\varphi} h, h^{*}>\right| \\
= & \left|<C_{\varphi}\left(h_{n}-h\right), h_{n}^{*}>\right| \\
& +\left|<C_{\varphi} h,\left(h_{n}^{*}-h^{*}\right)>\right| \\
\leq & \left\|C_{\varphi}\left(h_{n}-h\right)\right\|\left\|h_{n}^{*}\right\|
\end{aligned}
$$




$$
+\left|<C_{\varphi} h,\left(h_{n}^{*}-h^{*}\right)>\right|
$$

which converges to 0 since $C_{\varphi}$ is completely continuous and $h_{n} \rightarrow h$ weakly. Hence

$$
<C_{\varphi} h_{n}, h_{n}^{*}>\rightarrow\|h\|_{p}^{p}<C_{\varphi} \frac{h}{\|h\|_{p}}, \frac{h^{*}}{\left\|h^{*}\right\|_{q}}>
$$

as $n \rightarrow \infty$. Note that

$$
<C_{\varphi} \frac{h}{\|h\|_{p}}, \frac{h^{*}}{\left\|h^{*}\right\|_{q}}>\in W\left(C_{\varphi}\right)
$$

and from which we can conclude that $\bar{W}\left(C_{\varphi}\right) \subseteq \bigcup_{0 \leq \alpha \leq 1} \alpha W\left(C_{\varphi}\right)$. Now we show that $0 \in \bar{W}\left(C_{\varphi}\right)$. To see this let $\lambda \in U$ and note that $\left\|^{*} e_{\lambda}\right\|_{p}^{p}=\left\|e_{\lambda}\right\|_{q}^{q}=$ $\sum_{n=0}^{\infty} \frac{|\lambda|^{n q}}{\beta(n)^{q}}$. We have

$$
\begin{aligned}
<C_{\varphi}\left(\frac{{ }^{*} e_{\lambda}}{\left\|* e_{\lambda}\right\|_{p}}\right), \frac{e_{\lambda}}{\left\|e_{\lambda}\right\|_{q}}> & =\frac{1}{\left\|* e_{\lambda}\right\|_{p}\left\|e_{\lambda}\right\|_{q}}<{ }^{*} e_{\lambda}, C_{\varphi}^{*} e_{\lambda}> \\
& =\frac{1}{\left\|e_{\lambda}\right\|_{q}^{q}}<^{*} e_{\lambda}, e_{\varphi(\lambda)}> \\
& =\frac{1}{\left\|e_{\lambda}\right\|_{q}^{q}}{ }^{*} e_{\lambda}(\varphi(\lambda)) .
\end{aligned}
$$

Letting $\lambda \rightarrow \xi_{0}$, we get $0 \in \bar{W}\left(C_{\varphi}\right)$. Now if $W\left(C_{\varphi}\right)$ is closed, then $0 \in W\left(C_{\varphi}\right)$. Conversely, let $0 \in W\left(C_{\varphi}\right)$ and $0 \neq \alpha \in \bar{W}\left(C_{\varphi}\right)$. Then $\alpha \in c W\left(C_{\varphi}\right)$ for some $c \in(0,1]$. Since $W\left(C_{\varphi}\right)$ is convex and $0 \in W\left(C_{\varphi}\right)$, thus $\alpha \in W\left(C_{\varphi}\right)$ and so $\bar{W}\left(C_{\varphi}\right)=W\left(C_{\varphi}\right)$. Hence $W\left(C_{\varphi}\right)$ is closed and this completes the proof.

Corollary 2.5. Under the conditions of Theorem 2.4, $\bar{W}\left(C_{\varphi}\right)=W\left(C_{\varphi}\right) \cup$ $\{0\}$.

\section{References}

[1] P.S. Bourdon, J. H. Shapiro, When is zero in the numerical range of a composition operators?, Integer. Equ. Oper. Theory, 44 (2002), 410-441.

[2] B.D. MacCluer, X. Zong, N. Zorboska, Composition operators on small weighted Hardy spaces, Illinois Journal of Mathematics, 40 (4) (1996), 662-677.

[3] B. Yousefi, On the space $I^{p}(\beta)$, Rendiconti Del Circolo Mathmatico Di Palermo, Serie II, Tomo XILX (2000), 115-120.

[4] B. Yousefi, Bounded analytic structure of the Banach space of formal power series, Rendiconti Del Circolo Matematico Di Palermo, Serie II, Tomo LI (2002), 403-410. 
[5] B. Yousefi, S. Jahedi, Composition operators on Banach spaces of formal power series, Bollettino Della Unione Matematica Italiano, 8 (6-B) (2003), 481-487.

[6] B. Yousefi, A. I. Kashkuli, Cyclicity and unicellularity of the differentiation operator on Banach spaces of formal power series, Mathematical Proceedings of the Royal Irish Academy, 105A (1) (20050, 1-7.

[7] B. Yousefi, Sh. Khoshdel, Reflexivity of powers of the multiplication operator on special function spaces, Acta Mathematica Scientia, 32B (6) (2012), 2279-2284. 
